\title{
Biomechanical Evaluation of a Novel Posterior Integrated Clamp That Attaches to an Existing Posterior Instrumentation for Use in Thoracolumbar Revision
}

\author{
Patrick Senatus ${ }^{1}$, Suresh Reddy Chinthakunta ${ }^{2}$, Pedram Vazifeh ${ }^{2}$, Saif Khalil ${ }^{2}$ \\ ${ }^{1}$ University of Connecticut Health Center, Farmington, CT, USA \\ ${ }^{2}$ Globus Medical Inc., Audubon, PA, USA
}

Study Design: An in vitro biomechanical study.

Purpose: To evaluate the biomechanics of a novel posterior integrated clamp (IC) that extends on an already implanted construct in comparison to single long continuous bilateral pedicle screw (BPS) and rod stabilization system.

Overview of Literature: Revision surgery in the thoracolumbar spine often necessitates further instrumentation following a failed previous back surgery. Stability of these reconstructed constructs is not known.

Methods: Six osteoligamentous T12-L5 calf spines were tested on a spine motion simulator in the following configurations: intact, four level constructs (T13-L4), three level constructs (L1-L4), and two level constructs (L2-L4), by varying the ratio between BPS and IC. A load control protocol of $8 \mathrm{Nm}$ moments was applied at a rate of $1^{\circ} / \mathrm{sec}$ to establish the range of motion value for each construct in flexion-extension, lateral bending, and axial rotation. Statistical analysis was performed on raw data using repeated measures analysis of variance and significance was set at $p<0.05$.

Results: $0 \mathrm{n}$ an average, the reduction in motion for the four level continuous pedicle screw and rod construct (67\%) was similar to those extended with integrated clamps (64\%). Furthermore, for three level and two level constructs, no significant difference was observed between continuous pedicle screw constructs and those revised with the integrated clamps (regardless of the ratio between BPS and IC).

Conclusions: The novel posterior IC showed equivalent biomechanical rigidity to continuous pedicle screw rod constructs in revision scenarios. Clinical studies on posterior rod adjunct systems are necessary to confirm these results.

Keywords: Thoracic; Surgical revision; Bone screws; Internal fixation; Clamp

\section{Introduction}

Spinal surgeries have been successful in treating symptomatic spondylosis, but several patients still have postoperative symptoms that require single or multiple revision surgery [1]. The most common reasons for revision surgeries are pseudarthrosis, curve progression, infection, adjacent level degeneration, and implant failures

Received Mar 27, 2012; Revised Jul 7, 2012; Accepted Jul 7, 2012

Corresponding author: Suresh Reddy Chinthakunta

Globus Medical Inc., 2560 General Armistead Ave, Audubon, PA 19403, USA

Tel: +1-610-930-1800 (2502), Fax: +1-610-930-2042, E-mail: sureshoubme@gmail.com 
[2]. Lumbar spine surgery has been reported to have revision rates as high as $37 \%[1,3]$. It has been reported that within 3 to 5 years, $15 \%$ to $20 \%$ of patients undergoing fusion for degenerative conditions underwent a revision surgery due to significant back pain [4]. Improved surgical technique and a more careful way of patient selection can reduce the need for revision surgery; however, in many cases, it is not possible to avoid the need for longer posterior instrumentation [1]. More often than not, these revision surgeries require replacing the existing implants, which may increase the number of procedural steps, the length of the surgical incision, and the required operative time leading to further complications [5].

In the event of a revision surgery, the operating surgeon may either perform a complete implant removal or top off an extension system to the existing instrumentation. The latter is more common when treating adjacent instability while the former is usually required in cases of instrumentation failure. In the present study, we evaluated the biomechanics of a novel integrated clamp (IC) (Revere Addition Rescue, Globus Medical Inc., Audubon, PA, USA) extending on to the previously implanted pedicle screw rod construct. The system also includes novel implants used for extending an already implanted rigid pedicle screw rod system. Extension is accomplished by attaching an auxiliary rod to the last link of the previously implanted system with various connectors. Apart from a range of rod-to-rod connectors, the system includes contourable integrated clamps (Rescue, Globus Medical Inc.) (Fig. 1). Their single block design minimizes stress concentration at the rod-connection interface by a gradual radius and is mechanically stronger than a typical parallel connector. These specialized implants allow surgeries to be performed through a smaller incision with the potential for reduced surgical complications, decreased blood loss, and shorter operation time.

Use of novel integrated clamps in conjunction with screw and rod fixation system is of interest to many surgeons because of its ease-of-use and reduced procedural steps. For these advantages to be beneficial, the biomechanical properties of the system should be comparably stable than traditional internal rigid fixation. Hence, to accomplish this purpose, revision surgery was simulated with conventional bilateral pedicle screw (BPS) and the novel IC that extends onto an already implanted construct. In addition, the difference in biomechanical stability, if any, offered by this novel system was compared to a continuous BPS stabilization system by simulating various revision scenarios using a calf spine model (T12-L5).

\section{Materials and Methods}

\section{Specimen preparation}

A total of six thoracolumbar calf spines (T12-L5, 18-week-old) were used in the present study. Specimens were radiographed in the anterior-posterior and lateral planes to ensure the absence of any major anatomical abnormalities/fractures. The sacrum and L6 were removed in the slaughtering procedure. The spines were dissected by carefully denuding the paravertebral musculature, avoiding disruption of spinal ligaments, joints and disks. Each spine was potted proximally at T12 and distally at L5 in a 3:1 mixture of Bondo auto body filler (Bondo MarHyde Corp, Atlanta, GA, USA) and fiberglass resin (Home-Solution All Purpose Bondo MarHyde).

\section{Flexibility testing}

Each spine was fixed to the load frame of a custom-built six degree of freedom spine simulator and a pure moment was applied to the construct through servomotors [6]. The design of the load frame enables unconstrained motion of the spine in response to an applied load. There was no compressive preload applied on the specimen. A load control protocol was used to apply a maximum moment of $\pm 8 \mathrm{Nm}$ at a rate of $1 \% \mathrm{sec}[7,8]$. Three infrared light-

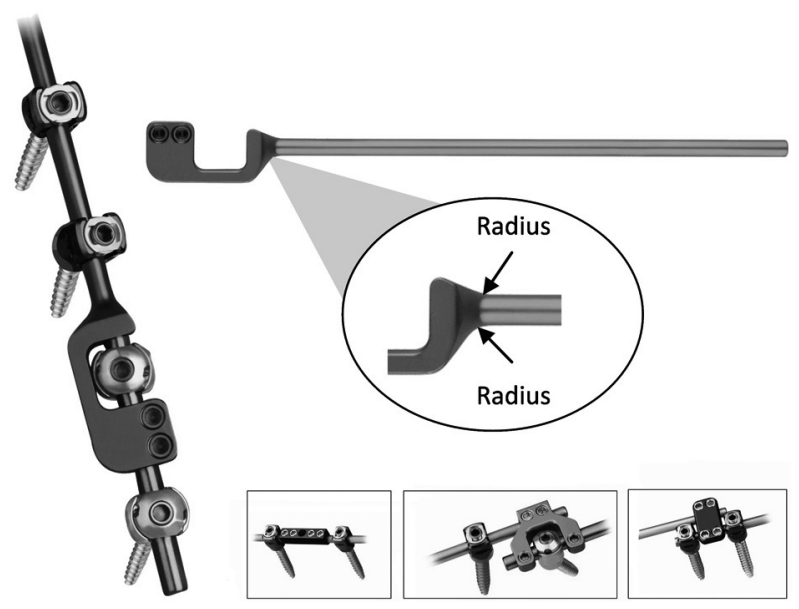

Fig. 1. Novel Integrated clamps connecting previously implanted bilateral pedicle screw instrumentation preventing the need to remove existing implant. Additional connectors available within the system that wed to a pre-existing longitudinal component. 
emitting diodes, mounted non-collinearly on a plexiglass plate were rigidly attached to the anterior aspect of each vertebral body T13, L1, L2, L3, and L4 and served as points for motion measurement. Three-dimensional motions were tracked using Optotrak Certus motion analysis system (NDI Inc., Waterloo, ON, Canada). Each of the test constructs were subjected to two load-unload cycles in each of the physiologic planes, thus generating flexionextension (FE), right-left lateral bending (LB), and rightleft axial rotation (AR) load displacement curves. The three dimensional intervertebral rotation was obtained from the motion analysis data files in the form of Euler angles (degrees) about the $\mathrm{X}, \mathrm{Y}$, and $\mathrm{Z}$-axes. The $\mathrm{R}_{\mathrm{x}} /-\mathrm{R}_{\mathrm{x}}$, $\mathrm{R}_{\mathrm{y}} /-\mathrm{R}_{\mathrm{y}}$, and $\mathrm{R}_{\mathrm{z}} /-\mathrm{R}_{\mathrm{z}}$ denotes $\mathrm{FE}, \mathrm{AR}$, and $\mathrm{LB}$ range of motion (ROM), respectively.

\section{Surgical constructs}

Each spine was initially tested in the intact state. Posterior instrumentation was then performed using BPS and rods using REVERE pedicle screws (Globus Medical Inc.) at T13-L4, L1-L4, and L2-L4 levels. Following posterior instrumentation, IC using Revere Addition Rescue clamps (Globus Medical Inc.) (Fig. 1) were instrumented
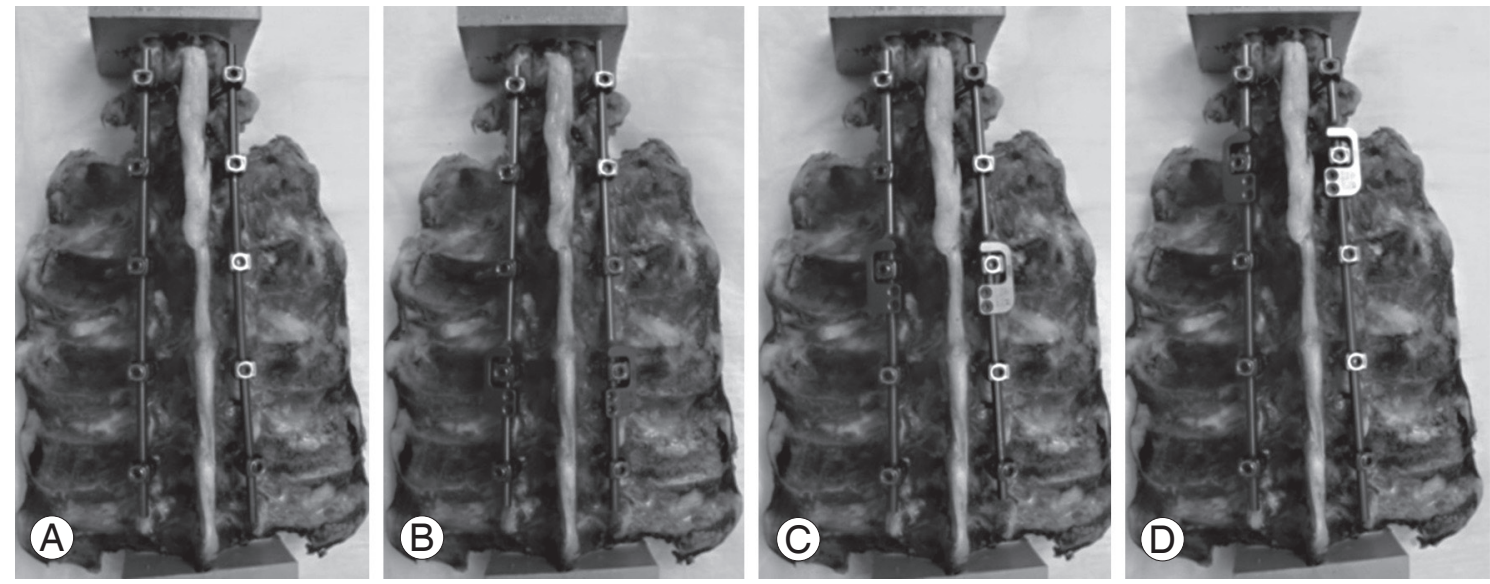

Fig. 2. Four level surgical constructs. (A) Bilateral pedicle screw (BPS) T13-L4, (B) BPS L3-L4+integrated clamp (IC) T13-L3, (C) BPS L2-L4+IC T13-L2, and (D) BPS L1-L4+IC T13-L1.
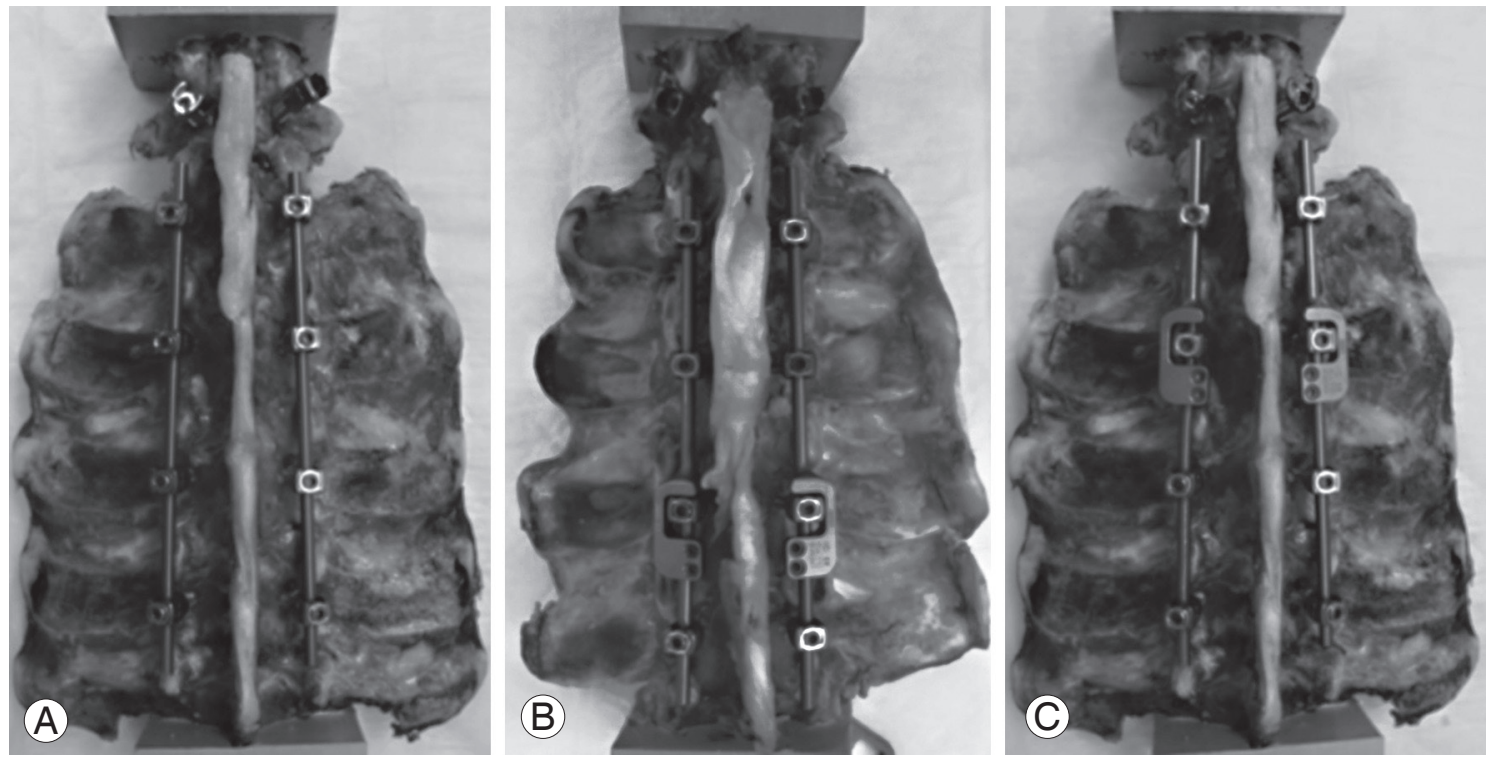

Fig. 3. Three level constructs. (A) Bilateral pedicle screw (BPS) L1-L4, (B) BPS L3-L4+integrated clamp (IC) L1-L3, (C) BPS L2-L4+IC L1-L2. 
Table 1. ROM values (degrees) for different surgical constructs at T13-L4

$\begin{array}{lccccc}\text { Loading condition } & \text { Intact } & \text { BPS T13-L4 } & \text { BPS L3-L4+IC T13-L3 } & \text { BPS L2-L4+IC T13-L2 } & \text { BPS L1-L4+IC T13-L1 } \\ \text { Flexion-extension } & 37 \pm 6 & 7 \pm 3 & 7 \pm 3 & 10 \pm 3 & 9 \pm 1 \\ \text { Lateral bending } & 58 \pm 11 & 17 \pm 4 & 17 \pm 4 & 21 \pm 6 & 21 \pm 5 \\ \text { Axial rotation } & 18 \pm 2 & 10 \pm 1 & 9 \pm 2 & 11 \pm 1 & 9 \pm 1\end{array}$

Values are presented as mean \pm standard deviation.

ROM, range of motion; BPS, bilateral pedicle screw; IC, integrated clamp.

adjacent to the BPS at T13-L4, L1-L4, and L2-L4 levels as shown in Figs. 2-4, simulating various revision scenarios.

Surgical constructs included: 1) Intact; 2) Four level constructs (Fig. 2): (1) BPS T13-L4, (2) BPS L3-L4+IC T13-L3 (1:3), (3) BPS L2-L4+IC T13-L2 (2:2), (4) BPS L1-L4+IC T13-L1 (3:1); 3) Three level constructs (Fig. 3): (1) BPS L1-L4, (2) BPS L3-L4+IC L1-L3 (1:2), (3) BPS L2-L4+IC L1-L2 (2:1); 4) Two level constructs (Fig. 4): (1) BPS L2-L4, (2) BPS L3-L4+IC L2-L3 (1:1).

\section{Data analysis}

Statistical analysis was performed on raw data using oneway repeated measures analysis of variance followed by Tukey's post-hoc for multiple comparison procedures. Significance was set at $p<0.05$.

\section{Results}

The constructs were examined after testing and showed no visible signs of damage, loosening, or breakage. ROM data were normalized to the intact spine (100\%) and reported as mean \pm standard deviation.

\section{T13-L4 ROM (four level constructs)}

Comparative ROM values for different surgical constructs are presented in Table 1. ROM for all the instrumented conditions reduced significantly in all loading modes when compared to the intact condition. There was no significant difference between the instrumented constructs for any of the loading conditions. The four level standard screw and rod construct reduced motion by $82 \%, 71 \%$, and $47 \%$ in FE, LB, and AR, respectively, when compared to the intact condition. Significance was achieved in $\mathrm{FE}$ and $\mathrm{LB}$. The 1:3 revision construct significantly reduced motion by $81 \%, 72 \%$, and $51 \%$, in $\mathrm{FE}, \mathrm{LB}$, and
AR, respectively. The $2: 2$ revision construct significantly resulted in $73 \%, 64 \%$ and $42 \%$ reduction in $\mathrm{FE}, \mathrm{LB}$, and $\mathrm{AR}$, respectively, when compared to the intact condition. Similarly, the 3:1 revision construct significantly reduced motion by $76 \%, 64 \%$, and $48 \%$ in $\mathrm{FE}, \mathrm{LB}$, and $\mathrm{AR}$, respectively when compared to the intact condition. ROM tended to increase minimally with $2: 2$ and $1: 3$ constructs.

\section{L1-L4 ROM (three level constructs)}

Comparative ROM values for the various surgical constructs are presented in Table 2. Mean ROM was significantly reduced in all the constructs and in all loading modes when compared to the intact condition. The continuous rod construct significantly reduced motion by $60 \%, 49 \%$, and $37 \%$ in $\mathrm{FE}, \mathrm{LB}$, and $\mathrm{AR}$, respectively, when compared to the intact condition. Furthermore, the $1: 2$ revision construct reduced motion by $61 \%, 50 \%$, and $40 \%$, in FE, LB, and AR, respectively, when compared to the intact condition. In a similar fashion, the 2:1 construct significantly reduced motion by $64 \%, 54 \%$, and $46 \%$ in FE, LB, and AR, respectively. No significant dif-
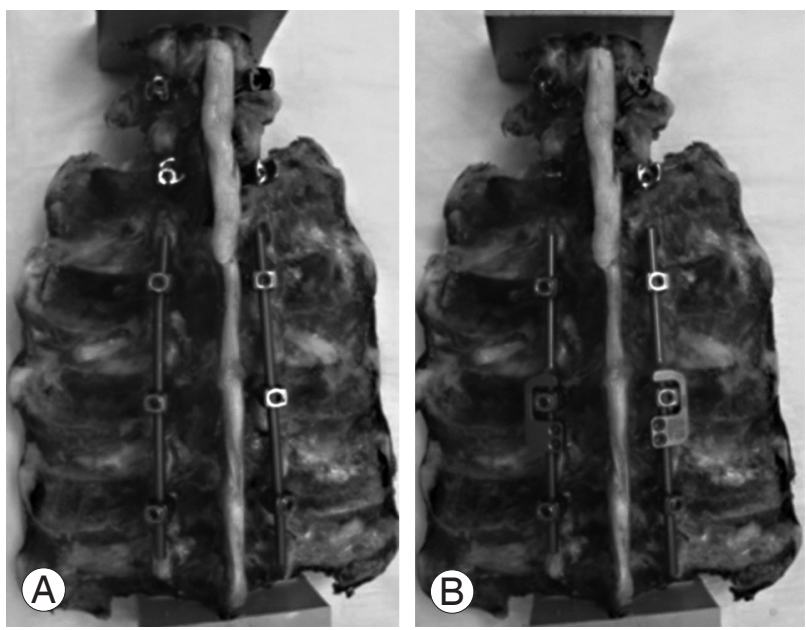

Fig. 4. Two level surgical constructs. (A) Bilateral pedicle screw (BPS) L2-L4 and (B) BPS L3-L4+integrated clamp L2-L3. 
Table 2. ROM values (degrees) for various surgical constructs at L1-L4

\begin{tabular}{lcccc}
\multicolumn{1}{c}{ Loading condition } & Intact & BPS L1-L4 & BPS L3-L4+IC L1-L3 & BPS L2-L4+IC L1-L2 \\
\hline Flexion-extension & $32 \pm 6$ & $13 \pm 2$ & $12 \pm 2$ & $12 \pm 2$ \\
\hline Lateral bending & $50 \pm 11$ & $26 \pm 5$ & $25 \pm 5$ & $23 \pm 4$ \\
\hline Axial rotation & $16 \pm 2$ & $10 \pm 1$ & $10 \pm 1$ & $9 \pm 2$ \\
\hline
\end{tabular}

Values are presented as mean \pm standard deviation.

ROM, range of motion; BPS, bilateral pedicle screw; IC, integrated clamp.

Table 3. ROM values (degrees) for tested surgical constructs at L2-L4

\begin{tabular}{lccc}
\multicolumn{1}{c}{ Loading condition } & Intact & BPS L2-L4 & BPS L3-L4+IC L2-L3 \\
Flexion-extension & $27 \pm 5$ & $16 \pm 1$ & $15 \pm 2$ \\
Lateral bending & $43 \pm 9$ & $28 \pm 3$ & $28 \pm 3$ \\
Axial rotation & $14 \pm 1$ & $10 \pm 1$ & $10 \pm 2$ \\
\hline
\end{tabular}

Values are presented as mean \pm standard deviation.

ROM, range of motion; BPS, bilateral pedicle screw; IC, integrated clamp.

ference in mean ROM was observed between the instrumented constructs for any of the loading conditions.

\section{L2-L4 ROM (two level constructs)}

Comparative mean ROM values for the tested surgical constructs are shown in Table 3. Upon comparison with the intact condition, mean ROM for all the instrumented conditions reduced significantly in FE, LB, and AR. There was no significant difference between the instrumented constructs in none of the loading conditions. The unrevised construct reduced motion significantly by $42 \%$, $36 \%$, and $27 \%$ in FE, $\mathrm{LB}$, and $\mathrm{AR}$, respectively. The 1:1 revision constructs reduced motion in a similar fashion.

\section{Discussion}

Reoperations requiring implant removal are not very exceptional. Implants are removed, although occasionally, following instrumented posterior spinal fusion due to late operative site pain and failed back surgery amongst several other factors [5]. In a retrospective case series of 185 patients, Asher et al. [9] reported an $8 \%$ reoperation rate for late operative site pain. In another retrospective study conducted by Gillet [10], 20\% of 78 patients followed for a minimum of 5 years required a secondary operation for extension of the fusion. Most of the lumbar revision surgeries involve replacing the existing implants, which may increase the number of procedural steps, the size of the surgical incision, and the operative time, possibly leading to further complications. In a retrospective cohort study consisting of 24,882 patients, Martin et al. [11] reported that $60 \%$ of reoperations following fusion were associated with either device complications or pseudoarthrosis. Deckey et al. [12] reported that patients with complete removal of the implant experienced increasing pain or collapse and Rathjen et al. [5] demonstrated that patients with solid fusion may have progressive spinal deformity following implant removal. These complications are, however, dependent on surgical treatment and instrumentation, which varies greatly depending upon the condition of the patient, the procedure originally performed, and the nature of the failure. The novel IC discussed in this paper was designed to minimize some of the complications of revision surgeries by simplifying the extension of existing constructs. However, the stability of such a system upon comparison with traditional BPS stabilization system is not known. Hence, this study was designed to test the biomechanical aspects (ROM) of the IC.

In the present study, three revision scenarios involving two, three, and four levels were simulated. First scenario compared the continuous BPS system (simulating a complete removal of the implant) to three fixations (1:3, 2:2, 3:1) with the IC as an add-on (simulating the revision scenarios). The next two revision scenarios were reproduced at two and three levels. The results of the study demonstrate that all the instrumented (four, three and two level) constructs significantly reduced the ROM of 
the spine when compared to the intact spine in all the loading modes. The results of this study also show that connecting rods, with up to a 3:1 ratio of extensional rods to rigid rods, provided equivalent biomechanical stability to a one piece rigid construct i.e., irrespective of the ratio of extensional rods to rigid rods the stability provided by the revised constructs was comparable to the continuous BPS system. The strength of the IC was in the radius (Fig. 1), the critical junction where the rod meets the clamp, which was mechanically stronger and lacked the stresses of a typical parallel connector. Therefore, it is proposed that adding an IC to an already existing instrumentation, where feasible, will not affect the stability of the system. This is especially useful when fusing the level above or below a previous fusion due to new radiculopathy or myelopathy in which decompression and extension of the earlier fusion is required. In such cases, instead of removing the complete fixation, the surgeon may add the IC adjacent to the existing posterior instrumentation, thereby minimizing the surgery time and amount of exposure. Thus, the novel IC discussed in this paper may be used as an extension stabilization system in revision scenarios to minimize complications associated with revision spine surgeries.

This study was carried out using calf spines. Previous biomechanical and anatomical studies have demonstrated similarities between calf and human lumbar spines with regard to physical and mechanical properties, establishing suitability as substitutes for human spines for in vitro studies [13-15]. In addition, it has previously been shown that the use of calf spines as models for human spines in the testing of implant stability is especially suitable if the parameter of primary interest is ROM $[14,15]$.

Often times in the thoracic region, pedicle screws are very close together and there may not be enough space for the application of novel IC. For such scenarios, additional connectors within the system (Fig. 1) can be used. These connectors avoid removal of proximal pedicle screws inevitable in certain revision cases. Such additional features of the system allow surgeries to be performed through a smaller incision with the potential for reduced surgical complications, blood loss, and operation time. The stability offered by these additional connectors was not evaluated in this study, and the study is limited in this regard. Furthermore, it would have been interesting to see the potential stress failure points of this novel IC using a finite element analysis. Perhaps in a future study this aspect will be investigated.

\section{Conclusions}

The use of novel IC adjacent to existing posterior instrumentation may be valuable to surgeons for a number of practical reasons, the most obvious being potentially reduced surgical time, and reduced invasiveness. This extending system placed adjacent to conventional BPS and rod system demonstrated equivalent biomechanical rigidity when compared to single long screw and rod construct. Clinical studies are required to confirm these findings.

\section{Conflict of interest}

No potential conflict of interest relevant to this article was reported.

\section{Acknowledgments}

The authors acknowledge funding for this project paid for by Globus Medical Inc. and was conducted using the machines and other equipment within their research department. Funding was provided in terms of research engineer salaries and was not directed to emphasized areas such as data analysis, manuscript writing, or specific avenues. The authors would like to thank Aditya Muzumdar for his assistance during testing.

\section{References}

1. Eichholz KM, Ryken TC. Complications of revision spinal surgery. Neurosurg Focus 2003;15:E1.

2. Pichelmann MA, Lenke LG, Bridwell KH, Good CR, O'Leary PT, Sides BA. Revision rates following primary adult spinal deformity surgery: six hundred forty-three consecutive patients followed-up to twenty-two years postoperative. Spine (Phila Pa 1976) 2010;35:219-26.

3. Frymoyer JW, Matteri RE, Hanley EN, Kuhlmann D, Howe J. Failed lumbar disc surgery requiring second operation. A long-term follow-up study. Spine (Phila Pa 1976) 1978;3:7-11.

4. Diwan AD, Parvartaneni H, Cammisa F. Failed degenerative lumbar spine surgery. Orthop Clin North Am 2003;34:309-24. 
5. Rathjen K, Wood M, McClung A, Vest Z. Clinical and radiographic results after implant removal in idiopathic scoliosis. Spine (Phila Pa 1976) 2007;32:21848.

6. Majid K, Chinthakunta S, Muzumdar A, Khalil S. A comparative biomechanical study of a novel integrated plate spacer for stabilization of cervical spine: an in vitro human cadaveric model. Clin Biomech (Bristol, Avon) 2012;27:532-6.

7. Goel VK, Panjabi MM, Patwardhan AG, Dooris AP, Serhan H; American Society for Testing and Materials. Test protocols for evaluation of spinal implants. J Bone Joint Surg Am 2006;88 Suppl 2:103-9.

8. Wilke HJ, Wenger K, Claes L. Testing criteria for spinal implants: recommendations for the standardization of in vitro stability testing of spinal implants. Eur Spine J 1998;7:148-54.

9. Asher M, Lai SM, Burton D, Manna B, Cooper A. Safety and efficacy of Isola instrumentation and arthrodesis for adolescent idiopathic scoliosis: two- to 12-year follow-up. Spine (Phila Pa 1976) 2004;29:2013-23.
10. Gillet P. The fate of the adjacent motion segments after lumbar fusion. J Spinal Disord Tech 2003;16:33845.

11. Martin BI, Mirza SK, Comstock BA, Gray DT, Kreuter W, Deyo RA. Reoperation rates following lumbar spine surgery and the influence of spinal fusion procedures. Spine (Phila Pa 1976) 2007;32:3827.

12. Deckey JE, Court C, Bradford DS. Loss of sagittal plane correction after removal of spinal implants. Spine (Phila Pa 1976) 2000;25:2453-60.

13. Cotterill PC, Kostuik JP, D’Angelo G, Fernie GR, Maki BE. An anatomical comparison of the human and bovine thoracolumbar spine. J Orthop Res 1986;4:298-303.

14. Wilke HJ, Krischak S, Claes LE. Formalin fixation strongly influences biomechanical properties of the spine. J Biomech 1996;29:1629-31.

15. Wilke HJ, Krischak ST, Wenger KH, Claes LE. Loaddisplacement properties of the thoracolumbar calf spine: experimental results and comparison to known human data. Eur Spine J 1997;6:129-37. 